\title{
Expectations for the high-energy neutrino detection from starburst galaxies with KM3NeT/ARCA
}

\section{Walid Idrissi Ibnsalih, ${ }^{a, c, *}$ Antonio Ambrosone,${ }^{a, b}$ Antonio Marinelli, ${ }^{a}$ Gennaro Miele, ${ }^{a, b, d}$ Pasquale Migliozzi, ${ }^{a}$ Ofelia Pisanti, ${ }^{a, b}$ and Ankur Sharma, ${ }^{e}$ on behalf of the KM3NeT Collaboration}

${ }^{a}$ INFN - Sezione di Napoli, Complesso Univ. Monte S. Angelo, I-80126 Napoli, Italy

bDipartimento di Fisica “Ettore Pancini”, Università degli studi di Napoli "Federico II”, Complesso Univ. Monte S. Angelo, I-80126 Napoli, Italy

“Università degli Studi della Campania "Luigi Vanvitelli”, Dipartimento di Matematica e Fisica, viale Lincoln 5, Caserta, 81100 Italy

${ }^{d}$ Scuola Superiore Meridionale, Università degli studi di Napoli "Federico II", Largo San Marcellino 10, 80138 Napoli, Italy

${ }^{e}$ Department of Physics and Astronomy, Uppsala University, Box 516, SE-75120 Uppsala, Sweden

Star-forming galaxies (SFGs) and starburst galaxies (SBGs) are extragalactic sources which could produce high-energy neutrinos. In principle, they could play a rather important role for explaining at least a sizeable part of IceCube's observations of astophysical neutrino. Using a recent theoretical model which implemented a blending of spectral indeces, we present the KM3NeT/ARCA sensitivities for such a diffuse flux from the startburst galaxies. In particular, we provide the 5-year differential sensitivity for the two building blocks of ARCA. We make use only of the track-like events in the range of $100 \mathrm{GeV}-10 \mathrm{PeV}$ differentiate in 11 bins of energy. We show how the upcoming neutrino telescope could observe the diffuse SFG and SBG within 5 years of data taking. We found the minimum of the sensitivity at around $100 \mathrm{TeV}$, which is also the energy where the SBG contribution is expected to peak. This would not only constrain the multi-component fit of the observed astrophysical neutrino flux at that energy $(100 \mathrm{TeV})$, but would also provide us a direct link between the star-forming activity in the reservoir environments and the hadronic emissions.

37th International Cosmic Ray Conference (ICRC 2021)

Berlin, Germany (Online),

12-23 July 2021

\footnotetext{
${ }^{*}$ Presenter
} 


\section{Introduction}

Astrophysical neutrinos have been measured by IceCube for more than a decade, although their origin is not known yet. Throughout the years, many constraints have been imposed to different source classes. For instance, the contribution of Galactic neutrinos is below $10 \%$ of IceCube's flux. The gamma-ray bursts have been constrained to contribute up to a few percent to the diffuse neutrino flux. Furthermore, the dedicated IceCube stacking analysis has constrained the contribution of resolved blazars to be less than $27 \%$ of the HESE neutrino flux (see also). Even the analyses carried out have found out that Star-forming and Starburst galaxies (SFGs and SBGs) cannot be responsible for the entire IceCube's measurements. As a result, investigating which source class contribute the most to IceCube observations is crucially important. In spite of all the limits, SFGs and SBGs are well-known extra-galactic sources which exhibit a strong star-forming activity. They are characterised by a high degree of turbulence, enhanced by a strong magnetic field $(B \sim 200 \mu G)$, which confine high-energy protons inside their core. This makes them suitable candidates to produce gamma-rays and neutrinos via hadronic collisions. Currently, only a dozen of these sources have been observed as point-like sources of gamma-rays by Fermi-LAT. In fact, their low gamma-ray luminosity makes it challenging to constrain the properties of their diffuse flux. Different authors have lately attempted to calculate their gamma-ray and neutrino fluxes, discovering that these particular sources could explain a big part of IceCube's observations. Furthermore, the IceCube collaboration has reported a $2.9 \sigma$ excess of neutrino events coming from NGC 1068, which is one of the SBG observed by the Fermi-LAT telescope. What is more, Ref. [1] has recently shown how the cores of SMC and Circinus galaxy could possibily be observed in six years of data taking by KM3NeT/ARCA, which would demonstrate that the star-forming activity is dominated by hadronic emissions. All of this means that future measurements along with upcoming neutrino telescopes could really provide us with remarkable insight into the contribution of SFGs and SBGs to the high-energy neutrino flux. In this context, we present a novel sensitivity study for the possible diffuse signal of this class of sources considering the phase 2.0 of the incoming KM3NeT/ARCA telescope. In particular, making use of the model put forward by [2], we calculate the sensitivity for different SBG scenarios between $100 \mathrm{GeV}$ and $10 \mathrm{PeV}$. The computation has been obtained by differentiating this energy range in 11 bins, taking into account up-going track-like events and introducing a Boosted Decision Tree (BDT) for the selection of the considered events. The Monte Carlo production used for this analysis accounts only for muonic and anti-muonic neutrinos interacting through Charge Current (CC). The proceeding is organised as follows: in Section 1, we outline the theoretical model we used, while, in section 2, we explain the characteristics of the KM3NeT/ARCA detector, in section 3, we explain the analysis we performed to calculate the sensitivity. In the end, in section 5, we comment on our results and draw our conclusions.

\section{Expected neutrino diffuse signal from Starburst galaxies}

In this section, we outline the model we followed to perform the forthcoming analysis. We utilise the model proposed by [2]. In particular, we suppose that the star-forming activity only happens in a small spherical region of the source, called Starburst Nucleus (SBN). We solve the 
leaky-box model equation in order to calculate the distribution of high-energy protons in the core of the galaxies.

$$
F_{p}=Q_{p}\left(\frac{1}{T_{a d v}}+\frac{1}{T_{\text {loss }}}+\frac{1}{T_{\text {diff } f}}\right)^{-1}
$$

where $F_{p}$ and $Q_{p}$ are respectively the distribution function and the injection rate for protons. The cosmic-ray distribution depends on the balance of different processes with characteristic timescales: the advection $\left(T_{a d v}\right)$, energy losses $\left(T_{\text {loss }}\right)$ and diffusion processes $\left(T_{\text {diff } f}\right)$ (see [2] for the details). We assume that protons are injected with a power-law spectrum in momentum space with spectral index $\alpha$. The protons have an exponential cutoff varying between $1-20 \mathrm{PeV}$, in agreement with [2]. The flux is normalized by requiring that each supernova releases into protons $10 \%$ of its total explosion kinetic energy $\left(10^{51} \mathrm{erg}\right)$. Neutrinos are emitted by the pion decay $\left(\pi \rightarrow \mu v_{\mu}, \mu \rightarrow e v_{e} v_{\mu}\right)$. We determine the neutrino flux using the approach given by [3], which assumes that the pions carry a fixed energy portion of the high-energy protons $\left(k_{\pi}=17 \%\right)$. To compute the diffuse neutrino flux, we exploit the high values of the star formation rate of SBGs. Indeed, their star-forming activity is highly likely to be a reliable tracer of their distribution in the Universe. Consequently, we use the method of the star formation rate function to constrain the number of starburst galaxies in the Universe. We consider the modified Shechter function $\operatorname{SSFR}(\mathrm{z}$, $\psi$ ), which has been obtained by fitting in the redshift interval $0 \leq z \leq 4.2$ the IR+UV data of a Herschel Source sample after subtracting the AGN contamination. Furthermore, we take advantage of the fact that SBGs are astrophysical reservoirs, which means that their non-thermal emissions are not mainly driven by their structural parameters, but rather on the star formation rate $(\psi)$, the spectral index $(\alpha)$ and the high-energy proton cut-off $\left(p_{\max }\right)$. Furthermore, as assumed by [2], we allow each SBG to have different values of $\alpha$ and we particularly use a Gaussian distribution for these parameters exploiting the experimental catalogue. The formula for the diffuse flux is [2]

$$
\begin{aligned}
\Phi_{v}^{\mathrm{SBG}}\left(E, p^{\mathrm{max}}\right)= & \int_{0}^{4.2} \mathrm{~d} z \int_{\psi_{*}}^{\infty} \mathrm{d} \log \psi \frac{c d_{c}(z)^{2}}{H(z)} \\
& \times \Phi_{\mathrm{SFR}}(z, \psi)\left\langle\phi_{\nu}\left(E, z, \psi, p^{\max }\right)\right\rangle_{\alpha},
\end{aligned}
$$

where $H(z)=H_{0} \sqrt{\Omega_{M}(1+z)^{3}+\Omega_{\Lambda}}$ is the Hubble parameter with $H_{0}=67.74 \mathrm{~km} \mathrm{~s}^{-1} \mathrm{Mpc}^{-1}$, $\Omega_{M}=0.31$ and $\Omega_{\Lambda}=0.69, \psi_{*}=2.6 \mathrm{M}_{\odot} \mathrm{yr}^{-1}$ and $\left\langle\phi_{\nu}\right\rangle_{\alpha}$ is the emitted neutrino fluxes averaged over the distribution of spectral indeces. The astrophysical flux provided by Eq. (2) peaks at hudrends of TeVs and it can explain up to $40 \%$ of the IceCube's observations without exceeding the gamma-ray constraints coming from the hadronic component of the extra-galatict backgound light (EGB) (see [2] for details). The behaviour of the flux is a more complex function than a simple power-law due to the spectral index blending. However, for energies greater than $100 \mathrm{GeV}$, it can be accommodated by a simple power-law with an exponential cut-off $\left(E_{c u t}\right)$. In particular, for the forth-coming analysis, we use the expression

$$
\Phi_{\nu}(E)=N \cdot\left(\frac{E}{100 \mathrm{GeV}}\right)^{-2} \cdot E^{-E / E_{c u t}}
$$


where $N=2.74 \cdot 10^{-12} \mathrm{GeV}^{-1} \mathrm{~cm}^{-2} \mathrm{~s}^{-1}$ and for $E_{\text {cut }}$ we use two different values: $0.2 \mathrm{PeV}$ and 0.5 $\mathrm{PeV}$ which respectively correspond to 5 and $12 \mathrm{PeV}$ for $p_{\max }$. These values are, in broad terms, consistent with the fact that neutrinos carry about $5 \%$ of the proton energy.

\section{KM3NeT-ARCA detector}

The KM3NeT is a neutrino telescope in the construction phase, which will be located in the Mediterranean Sea, with the main purpose to search for high-energy neutrinos originated from Galactic and extragalactic diffuse and point-like sources. The KM3NeT project actually consists of two three-dimensional Cherenkov neutrino detectors built with the same technology but with different dimensions: Oscillations Research with Cosmics in the Abyss (ORCA) and Astroparticle Research with Cosmics in the Abyss (ARCA). The ORCA detector will be installed at a sea bottom depth of about $2500 \mathrm{~m}$ near Toulon. The main purpose of ORCA detector is to study fundamental properties of neutrinos (neutrino mass ordering, oscillations etc.) and for this reason this detector is designed to detect neutrinos in the energy range 1-100 GeV. Instead the main goal of ARCA detector, installed at a sea bottom depth of about 3500 meters near Capo Passero (Sicily), is to observe the astrophysical neutrino flux and to identify possible sources of high-energy neutrinos. Therefore, the ARCA detector is optimised to have a maximum detection efficiency in the neutrino energy 1 $\mathrm{TeV}-10 \mathrm{PeV}$. As previously reported, the two KM3NeT detectors share the same technology, but they have different instrumentation densities due to distinct primary physics goals. The detector consists of the so-called Digital Optical Modules (DOMs): glass spheres resistant to water pressure instrumented with 31 photomultiplier tubes (PMTs), in order to detect the Cherenkov photons. A vertical sequence of DOMs forms a so-called Detection Unit (DU). The ARCA detector is a $\mathrm{km}^{3}$-sized detector: it will be built with a vertical spacing of 36 meters between the DOMs and a $90 \mathrm{~m}$ horizontal distance between the DUs. Currently six DUs have been installed for ARCA (this configuration is referred to as ARCA6) and are in data taking since May 2021.

\section{ARCA differential sensitivity for the SBGs diffuse signal}

In order to obtain a prediction on the possibility to observe the diffuse signal from SBGs sources with the KM3NeT-ARCA detector, the calculation of an appropriate sensitivity was performed (defined as in [4]). Given a model flux $\Phi_{s}$ (see equation 3, where in our case $N=2.74 \cdot 10^{-12}$ $\mathrm{GeV}^{-1} \mathrm{~cm}^{-2} \mathrm{~s}^{-1}$ and $E_{\text {cut }}=0.5$ ), the sensitivity is calculated at $90 \%$ of Confidence Level (CL) median upper limit following the Neyman method [5]):

$$
\Phi_{90}=\Phi_{s} \cdot \frac{n_{90}}{n_{s}}
$$

where $n_{s}$ is the mean number of signal events and $n_{90}$ is the number of events needed to obtain $P\left(N_{s}\right)=0.9$. Thus the sensitivity is referred to a particular flux that can be excluded at a $90 \%$ C.L. (in this analysis), if no significant signal is observed.

In this analysis the background was composed by the atmospheric muons and neutrinos. The sensitivity was calculated using the latest version of the KM3NeT-ARCA115 MC simulation:

- v5.2 for atmospheric muon. 
- v5.1 for atmospheric neutrino.

- v5.1 for signal SBGs.

Furthermore we used the same method to simulate atmospheric neutrinos and diffuse neutrino signal (from SBGs). However for the case of diffuse signal we renormalized the neutrino spectrum for the expected diffuse SED of SGBs following the already cited model.

The sensitivity was obtained by considering only muons from $v_{\mu}$ and $\bar{v}_{\mu}$ charge-current interactions for both the signal and atmospheric neutrinos.

The calculation was performed with several considerations:

- A total energy interval between $100 \mathrm{GeV}$ and $10 \mathrm{PeV}$ was considered: differentiating the sensitivity calculation in 11 bins of energy (log scale was considered).

- No selection in declination was performed.

- Two building block (BB) of KM3NeT-ARCA detector were taken into account.

- The sensitivity at $90 \%$ C.L. is computed for an observational time of five years.

The optimization of this study was obtained by applying event quality cuts for each of the 11 bins of energy considered; in details the following selections have been applied:

- A pre-selection cut in the recostructed angle is applied in order to reject the background.

- A multivariate analysis involving machine learning is performed to distinguish the background events from the SBG neutrinos.

On this regard for the atmospheric muons rejection, for each energy bin as a first selection comprising only events with a reconstructed angle $\theta_{\text {rec }}<100$ (up-going events). Furthermore to improve the rejection of background events, a dedicated machine learning tool was used. This tool includes the scan of some of the variables characterizing the reconstructed events (reported in table 1), is based on a BDT algorithm to decide if an event is classified as a "signal-like" or "background-like". The algorithm was implemented from the ROOT:TMVA framework: for this particular analysis a Gradient boosting was used.

Table 1: A list of variables used to build the BDT algorithm: the energy reconstructed, best likelihood, track length, number of hits, number of photo-electron, $\beta_{0}$ angular error and ToT (Time-over-threshold). The algorithm used to reconstruct the tracks events is JGandalf.

\begin{tabular}{|c|c|}
\hline Variable & Description \\
\hline \hline$E_{\text {rec }}$ & Energy reconstructed with the track reconstruction algorithm \\
\hline Lik & Likelihood from track reconstruction algorithm \\
\hline length & Distance between first and last hits in metres \\
\hline n.hits & Number of hits used in the track recontruction algorithm \\
\hline npe & Number of photo-electrons along the whole track \\
\hline$\beta_{0}$ & Angular error from track reconstruction algorithm \\
\hline ToT & Sum of the ToT for hits used for the track reconstruction \\
\hline
\end{tabular}


Once we obtained the output BDT-score classifier for each event, then it was used as a cut variable with the following assumptions:

- Events with $\theta_{\text {rec }}<80^{\circ}$ were selected with a BDT-score $>0.1$

- Events with $80^{\circ}<\theta_{\text {rec }}<100^{\circ}$ were selected with a BDT-score $>0.7$

The number of events reconstructed (in 1 year) bin-per-bin for signal and background before and after the selection performed are reported in the tables 2 and 3:

Table 2: Here are reported the reconstructed events bin-per-bin (interval range $100 \mathrm{GeV}-10 \mathrm{PeV}$ ) respectively for background and signal before the selection chain was applied.

\begin{tabular}{|cccc|}
\hline Interval energy & Atmospheric muon & Atmospheric neutrino & Signal \\
\hline \hline $10^{2} \mathrm{GeV}$ & 175002.2 & 10917.0 & 24.47 \\
$10^{2.5} \mathrm{GeV}$ & 303931.4 & 29031.8 & 53.0 \\
$10^{3} \mathrm{GeV}$ & 877022.0 & 26660.6 & 141.2 \\
$10^{3.5} \mathrm{GeV}$ & 5347572.6 & 12061.6 & 169.9 \\
$10^{4} \mathrm{GeV}$ & 29622046.0 & 3176.6 & 142.8 \\
$10^{4.5} \mathrm{GeV}$ & 30205346.3 & 519.4 & 90.0 \\
$10^{5} \mathrm{GeV}$ & 4762621.1 & 58.1 & 37.5 \\
$10^{5.5} \mathrm{GeV}$ & 203797.1 & 8.7 & 10.1 \\
$10^{6} \mathrm{GeV}$ & 35751.7 & 0.3 & 1.5 \\
$10^{6.5} \mathrm{GeV}$ & 16214.8 & 0.0184 & 0.13 \\
$10^{7} \mathrm{GeV}$ & 7942.9 & 0.00064 & 0.02 \\
\hline
\end{tabular}

Table 3: Here are reported the reconstructed events bin-per-bin (interval energy $100 \mathrm{GeV}-10 \mathrm{PeV}$ ) respectively for background and signal after the selection chain (with BDT) was applied.

\begin{tabular}{|cccc|}
\hline Interval energy & Atmospheric muon & Atmospheric neutrino & Signal \\
\hline \hline $10^{2} \mathrm{GeV}$ & 3.36 & 4683.0 & 15.4 \\
$10^{2.5} \mathrm{GeV}$ & 37.1 & 12150.8 & 34.3 \\
$10^{3} \mathrm{GeV}$ & 41.5 & 11397.2 & 75.8 \\
$10^{3.5} \mathrm{GeV}$ & 74.2 & 3137.2 & 91.7 \\
$10^{4} \mathrm{GeV}$ & 48.8 & 411.7 & 67.7 \\
$10^{4.5} \mathrm{GeV}$ & 33.3 & 38.6 & 35.6 \\
$10^{5} \mathrm{GeV}$ & 0.0 & 20.3 & 13.2 \\
$10^{5.5} \mathrm{GeV}$ & 0.0 & 1.08 & 5.2 \\
$10^{6} \mathrm{GeV}$ & 0.0 & 0.03 & 0.35 \\
$10^{6.5} \mathrm{GeV}$ & 0.0 & 0.0085 & 0.012 \\
$10^{7} \mathrm{GeV}$ & 0.0 & 0.0 & 0.0 \\
\hline
\end{tabular}

In the table $\mathrm{n}$. 4 we report also the signal and background reconstructed events considering the entire energy interval $(100 \mathrm{GeV}-10 \mathrm{PeV})$ : 
Table 4: Here are reported the total reconstructed events respectively for background and signal before and after the selection chain applied (considering the entire interval energy).

\begin{tabular}{|c|c|c|c|}
\hline Type & Reconstructed events & Up going selection & BDT cut \\
\hline \hline Atmospheric muon & 35336090.0 & 369340.0 & 238.4 \\
\hline Atmospheric neutrino & 80431.7 & 60186.6 & 31854.9 \\
\hline Signal SBGs & 670.1 & 405.5 & 337.4 \\
\hline
\end{tabular}

Definitely once we applied all the selection chain we calculated bin-per-bin the sensitivity by the formula 4. The differential KM3NeT/ARCA sensitivity for the described model of SBGs in equation 3 is shown in fig. 1. In this plot we report for comparison also the full sky diffuse flux obtained by IceCube (reported with grey points) through HESE and CASCADE samples. In fig. 1, it is shown a comparison between the calculated differential sensitivity and the the models constrained by the diffuse data of gamma-ray (EGB) and neutrino (HESE and CASCADE samples).
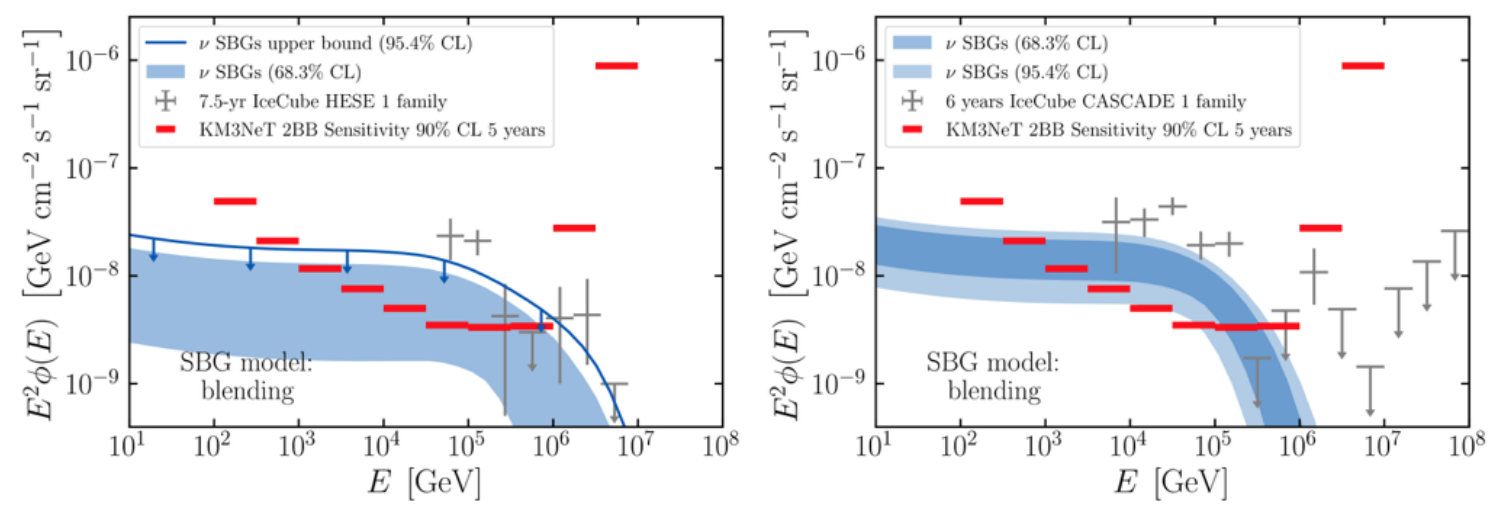

Figure 1: On the left is reported the differential sensitivity at $90 \%$ of confidence level, between $100 \mathrm{GeV}-10$ $\mathrm{PeV}$ and for 5 years of KM3NeT/ARCA (2BB), compared to the diffuse emission expected from Starburst galaxies following [2]. The eleven bins of energy has been reported in order to highlight the region of the spectrum where the experiment is more sensitive. The upper blue line represents the model upper limit (1 sigma parameter space) taking into account the bounds coming from the multi-component fitting of the EGB measurements of Fermi-LAT and the HESE [6] sample measured by IceCube. The blue shadowed region indicates the SED expected from this class of sources considering the possible value within 2 sigma region of the Starburst blending scenario. The HESE spectral measurements are reported as a reference of the total observed diffuse astrophysical flux. On the right we reported the differential sensitivity at $90 \%$ C.L. with the same conditions (in $100 \mathrm{GeV}-10 \mathrm{PeV}$ for $2 \mathrm{BB}$ and 5 years) to the diffuse emission expected from Starburst galaxies following [2]. In this plot the blue shadowed region indicates the SED expected from this class of sources considering the possible value within 1 and 2 sigma regions of the Starburst blending scenario when the measurements of EGB from Fermi-LAT and the CASCADE sample [7] from IceCube are taken into account. The CASCADE spectral measurements are reported as a reference of the total observed diffuse astrophysical flux.

\section{Discussion and Conclusions}

Fig. 1 compares the differential sensitivity we obtained with both the theoretical expected Starburst SED and IceCube data samples (respectively HESE and CASCADES). Remarkably, we 
found that the expected SBG signal can potentially be observed by KM3NeT/ARCA (2BB) in 5 years of data taking. In fact, the minimum of the sensitivity resides in the region of hundreds of TeVs, namely where the SBG spectral energy distribution (SED) is expected to peak. On the other hand, the abrupt surge in the differential sensitivity above $10^{5-6} \mathrm{GeV}$ is due to the fact that the SFG and SBG neutrino signal is exponentially suppressed by the cut-off ( 200 - 500 $\mathrm{TeV}$ ). For comparison we report also the diffuse IceCube observations even though we did not performed the calculation of the sensitivity using IceCube's flux, which is well accommodated by an un-broken power-law, but rather using the physical signal expected from SFGs and SBGs. Our result is highly significant since a detection of the expected SBG signal from KM3NeT/ARCA would confirm the fact that this particular source class could produce a sizeable contribution at $100 \mathrm{TeV}$. Furthermore, this would not only demonstrate that the star-forming activity is directly linked to hadronic emissions but it would also give stronger constraints on the full sky contribution of this source class. However, one of the drawbacks of our analysis resides in the fact that, in order to explain a big part of IceCube's measurements with Starburst Galaxies, we need also to take into account the deep Universe $(z \sim 4-5)$ [2]. Therefore, it will be hard to disentangle the contribution coming from the "near Universe", namely coming from the known Star-forming and Starburst Galaxies with the contribution coming from the unresolved galaxies. On the whole, our work demonstrate how important SFGs and SBGs might be for the high-energy neutrino production and that the next decade of upcoming KM3NeT/ARCA will be a crucial to constrain their effective contribution with respect to IceCube's measurements. Indeed, the advent of a Global Neutrino Network would be necessary to unveil the origin of the high-energy astrophysical neutrinos and open a new era for the multi-messenger astronomy.

\section{References}

[1] Antonio Ambrosone, Marco Chianese, Damiano F. G. Fiorillo, Antonio Marinelli, and Gennaro Miele. Could nearby star-forming galaxies light up the point-like neutrino sky? 62021.

[2] Antonio Ambrosone, Marco Chianese, Damiano F. G. Fiorillo, Antonio Marinelli, Gennaro Miele, and Ofelia Pisanti. Starburst galaxies strike back: a multi-messenger analysis with Fermi-LAT and IceCube data. Mon. Not. Roy. Astron. Soc., 503(3):4032-4049, 2021.

[3] S.R. Kelner, Felex A. Aharonian, and V.V. Bugayov. Energy spectra of gamma-rays, electrons and neutrinos produced at proton-proton interactions in the very high energy regime. Phys. Rev. D, 74:034018, 2006. [Erratum: Phys.Rev.D 79, 039901 (2009)].

[4] S. Aiello et al. Sensitivity of the KM3NeT/ARCA neutrino telescope to point-like neutrino sources, arXiv:1107.1593v1. Astroparticle physics, 2019.

[5] J. Neyman. Phil. Trans. Roy. Soc. A, 236, p. 333. 1937.

[6] The IceCube Collaboration. Characterization of the astrophysical diffuse neutrino flux with icecube high-energy starting events. 2019.

[7] M. G. Aartsen. Search for Astrophysical Sources of Neutrinos Using Cascade Events in IceCube. The Astrophysical Journal, 846:136 (12pp), 2017. 


\section{The KM3NeT Collaboration author list (July 2021)}

M. Ageron ${ }^{1}$, S. Aiello ${ }^{2}$, A. Albert ${ }^{3,55}$, M. Alshamsi ${ }^{4}$, S. Alves Garre ${ }^{5}$, Z. Aly ${ }^{1}$, A. Ambrosone ${ }^{6,7}$, F. Ameli ${ }^{8}$, M. Andre 9 , G. Androulakis ${ }^{10}$, M. Anghinolfi ${ }^{11}$, M. Anguita ${ }^{12}$, G. Anton ${ }^{13}$, M. $\operatorname{Ardid}^{14}$, S. $\operatorname{Ardid}^{14}$, W. Assal ${ }^{1}$, J. Aublin ${ }^{4}$, C. Bagatelas ${ }^{10}$, B. Baret ${ }^{4}$, S. Basegmez du Pree ${ }^{15}$, M. Bendahman ${ }^{4,16}$, F. Benfenati ${ }^{17,18}$, E. Berbee ${ }^{15}$, A. M. van den Berg ${ }^{19}$, V. Bertin ${ }^{1}$, S. Beurthey ${ }^{1}$, V. van Beveren ${ }^{15}$, S. Biagi ${ }^{20}$, M. Billault ${ }^{1}$, M. Bissinger ${ }^{13}$, M. Boettcher ${ }^{21}$, M. Bou Cabo ${ }^{22}$, J. Boumaaza ${ }^{16}$, M. Bouta ${ }^{23}$, C. Boutonnet ${ }^{4}$, G. Bouvet ${ }^{24}$, M. Bouwhuis ${ }^{15}$, C. Bozza ${ }^{25}$, H.Brânzass ${ }^{26}$, R. Bruijn ${ }^{15,27}$, J. Brunner ${ }^{1}$, R. Bruno ${ }^{2}$, E. Buis ${ }^{28}$, R. Buompane ${ }^{6,29}$, J. Busto ${ }^{1}$, B. Caiffi ${ }^{11}$, L. Caillat ${ }^{1}$, D. Calvo ${ }^{5}$, S. Campion ${ }^{30,8}$, A. Capone ${ }^{30,8}$, H. Carduner ${ }^{24}$, V. Carretero ${ }^{5}$, P. Castaldi ${ }^{17,31}$, S. Celli ${ }^{30,8}$, R. Cereseto ${ }^{11}$, M. Chabab ${ }^{32}$, C. Champion ${ }^{4}$, N. $\mathrm{Chau}^{4}$, A. Chen ${ }^{33}$, S. Cherubinini ${ }^{20,34}$, V. Chiarella ${ }^{35}$, T. Chiarusi ${ }^{17}$, M. Circella ${ }^{36}$, R. Cocimano ${ }^{20}$, J. A. B. Coelho ${ }^{4}$, A. Coleiro ${ }^{4}$, M. Colomer Molla ${ }^{4,5}$, S. Colonges ${ }^{4}$, R. Coniglione ${ }^{20}$, A. Cosquer ${ }^{1}$, P. Coyle ${ }^{1}$, M. Cresta ${ }^{11}$, A. Creusot ${ }^{4}$, A. $\mathrm{Cruz}^{37}$, G. Cuttone ${ }^{20}$, A. D’Amico ${ }^{15}$, R. Dallier ${ }^{24}$, B. De Martino ${ }^{1}$, M. De Palma ${ }^{36,38}$, I. Di Palma ${ }^{30,8}$, A. F. Díaz ${ }^{12}$, D. DiegoTortosa $^{14}$, C. Distefano ${ }^{20}$, A. Domi ${ }^{15,27}$, C. Donzaud ${ }^{4}$, D. Dornic ${ }^{1}$, M. Dörr ${ }^{39}$, D. Drouhin ${ }^{3,55}$, T. Eberl ${ }^{13}$, A. Eddyamoui ${ }^{16}$, T. van Eeden ${ }^{15}$, D. van Eijk ${ }^{15}$, I. El Bojaddaini ${ }^{23}$, H. Eljarrari ${ }^{16}$, D. Elsaesser ${ }^{39}$, A. Enzenhöfer ${ }^{1}$, V. Espinosa ${ }^{14}$, P. Fermani ${ }^{30,8}$, G. Ferrara ${ }^{20,34}$, M. D. Filipović ${ }^{40}$, F. Filippini ${ }^{17,18}$, J. Fransen ${ }^{15}$, L. A. Fusco ${ }^{1}$, D. Gajanana ${ }^{15}$, T. Gal ${ }^{13}$, J. García Méndez ${ }^{14}$, A. Garcia Soto $^{5}$, E. Garçon ${ }^{1}$, F. Garufi ${ }^{6,7}$, C. Gatius ${ }^{15}$, N. Geißelbrecht ${ }^{13}$, L. Gialanella ${ }^{6,29}$, E. Giorgio ${ }^{20}$, S. R. Gozzini ${ }^{5}$, R. Gracia ${ }^{15}$, K. Graf ${ }^{13}$, G. Grella ${ }^{41}$, D. Guderian ${ }^{56}$, C. Guidi ${ }^{11,42}$, B. Guillon ${ }^{43}$, M. Gutiérrez ${ }^{44}$, J. Haefner ${ }^{13}$, S. Hallmann ${ }^{13}$, H. Hamdaoui ${ }^{16}$, H. van Haren ${ }^{45}$, A. Heijboer ${ }^{15}$, A. Hekalo ${ }^{39}$, L. Hennig ${ }^{13}$, S. Henry ${ }^{1}$, J. J. Hernández-Rey ${ }^{5}$, J. Hofestädt ${ }^{13}$, F. Huang ${ }^{1}$, W. Idrissi Ibnsalih ${ }^{6,29}$, A. Ilioni ${ }^{4}$, G. Illuminati ${ }^{17,18,4}$, C. W. James ${ }^{37}$, D. Janezashvili ${ }^{46}$, P. Jansweijer ${ }^{15}$, M. de Jong ${ }^{15,47}$, P. de Jong ${ }^{15,27}$, B. J. Jung ${ }^{15}$, M. Kadler ${ }^{39}$, P. Kalaczyński ${ }^{48}$, O. Kalekin ${ }^{13}$, U. F. Katz ${ }^{13}$, F. Kayzel ${ }^{15}$, P. Keller ${ }^{1}$, N. R. Khan Chowdhury ${ }^{5}$, G. Kistauri ${ }^{46}$, F. van der Knaap ${ }^{28}$, P. Kooijman ${ }^{27,57}$, A. Kouchner ${ }^{4,49}$, M. Kreter ${ }^{21}$, V. Kulikovskiy ${ }^{11}$, M. Labalme ${ }^{43}$, P. Lagier ${ }^{1}$, R. Lahmann ${ }^{13}$, P. Lamare ${ }^{1}$, M. Lamoureux ${ }^{14}$, G. Larosa ${ }^{20}$, C. Lastoria ${ }^{1}$, J. Laurence ${ }^{1}$, A. Lazo $^{5}$, R. Le Breton ${ }^{4}$, E. Le Guirriec ${ }^{1}$, S. Le Stum ${ }^{1}$, G. Lehaut ${ }^{43}$, O. Leonardi ${ }^{20}$, F. Leone ${ }^{20,34}$, E. Leonora ${ }^{2}$, C. Lerouvillois ${ }^{1}$, J. Lesrel ${ }^{4}$, N. Lessing ${ }^{13}$, G. Levi ${ }^{17,18}$, M. Lincetto ${ }^{1}$, M. Lindsey Clark ${ }^{4}$, T. Lipreau ${ }^{24}$, C. LLorens Alvarez ${ }^{14}$, A. Lonardo ${ }^{8}$, F. Longhitano ${ }^{2}$, D. Lopez-Coto ${ }^{44}$, N. Lumb ${ }^{1}$, L. Maderer ${ }^{4}$, J. Majumdar ${ }^{15}$, J. Mańczak ${ }^{5}$, A. Margiotta ${ }^{17,18}$, A. Marinelli ${ }^{6}$, A. Marini ${ }^{1}$, C. Markou $^{10}$, L. Martin ${ }^{24}$, J. A. Martínez-Mora ${ }^{14}$, A. Martini ${ }^{35}$, F. Marzaioli ${ }^{6,29}$,

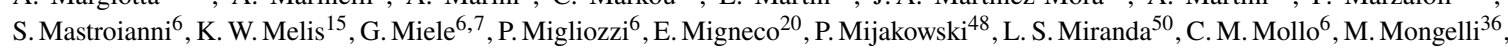
A. Moussa ${ }^{23}$, R. Muller ${ }^{15}$, P. Musico ${ }^{11}$, M. Musumeci ${ }^{20}$, L. Nauta ${ }^{15}$, S. Navas ${ }^{44}$, C. A. Nicolau ${ }^{8}$, B. Nkosi ${ }^{33}$, B. Ó Fearraigh ${ }^{15,27}$, M. O'Sullivan ${ }^{37}$, A. Orlando ${ }^{20}$, G. Ottonello ${ }^{11}$, S. Ottonello ${ }^{11}$, J. Palacios González ${ }^{5}$, G. Papalashviliit ${ }^{46}$, R. Papaleo ${ }^{20}$, C. Pastore ${ }^{36}$, A. M. Păun ${ }^{26}$, G.E. Păvălaşş, G. Pellegrinin ${ }^{17}$, C. Pellegrino ${ }^{18,58}$, M. Perrin-Terrini ${ }^{1}$, V. Pestel ${ }^{15}$, P. Piattelli ${ }^{20}$, C. Pieterse ${ }^{5}$, O. Pisanti ${ }^{6,7}$, C. Poirè ${ }^{14}$, V. Popa $^{26}$, T. Pradier $^{3}$, F. Pratolongo ${ }^{11}$, I. Probst ${ }^{13}$, G. Pühlhofer ${ }^{51}$, S. Pulvirenti ${ }^{20}$, G. Quéméner ${ }^{43}$, N. Randazzo ${ }^{2}$, A. Rapicavoli ${ }^{34}$, S. Razzaque $^{50}$, D. Real ${ }^{5}$, S. Reck ${ }^{13}$, G. Riccobene ${ }^{20}$, L. Rigalleau ${ }^{24}$, A. Romanov ${ }^{11,42}$, A. Rovelli ${ }^{20}$, J. Royon ${ }^{1}$, F. Salesa Greus ${ }^{5}$, D. F. E. Samtleben ${ }^{15,47}$, A. Sánchez Losa ${ }^{36,5}$, M. Sanguineti ${ }^{11,42}$, A. Santangelo ${ }^{51}$, D. Santonocito ${ }^{20}$, P. Sapienza ${ }^{20}$, J. Schmelling ${ }^{15}$, J. Schnabel ${ }^{13}$, M. F. Schneider ${ }^{13}$, J. Schumann ${ }^{13}$, H. M. Schutte ${ }^{21}$, J. Seneca ${ }^{15}$, I. Sgura ${ }^{36}$, R. Shanidze ${ }^{46}$, A. Sharma ${ }^{52}$, A. Sinopoulou ${ }^{10}$, B. Spisso ${ }^{41,6}$, M. Spurio ${ }^{17,18}$, D. Stavropoulos ${ }^{10}$, J. Steijger ${ }^{15}$, S. M. Stellacci ${ }^{41,6}$, M. Taiuti ${ }^{11,42}$, F. Tatone ${ }^{36}$, Y. Tayalati ${ }^{16}$, E. Tenllado ${ }^{44}$, D. Tézier ${ }^{1}$, T. Thakore ${ }^{5}$, S. Theraube ${ }^{1}$, H. Thiersen $^{21}$, P. Timmer $^{15}$, S. Tingay ${ }^{37}$, S. Tsagkli $^{10}$, V. Tsourapis ${ }^{10}$, E. Tzamariudaki ${ }^{10}$, D. Tzanetatos ${ }^{10}$, C. Valieri ${ }^{17}$, V. Van Elewyck ${ }^{4,49}$, G. Vasileiadis ${ }^{53}$, F. Versari ${ }^{17,18}$, S. Viola ${ }^{20}$, D. Vivolo ${ }^{6,29}$, G. de Wasseige ${ }^{4}$, J. Wilms $^{54}$, R. Wojaczyński ${ }^{48}$, E. de Wolf ${ }^{15,27}$, T. Yousfi ${ }^{23}$, S. Zavatarelli ${ }^{11}$, A. Zegarelli ${ }^{30,8}$, D. Zito ${ }^{20}$, J. D. Zornoza ${ }^{5}$, J. Zúñiga ${ }^{5}$, N. Zywucka ${ }^{21}$.

${ }^{1}$ Aix Marseille Univ, CNRS/IN2P3, CPPM, Marseille, France.

${ }^{2}$ INFN, Sezione di Catania, Via Santa Sofia 64, Catania, 95123 Italy.

${ }^{3}$ Université de Strasbourg, CNRS, IPHC UMR 7178, F-67000 Strasbourg, France.

${ }^{4}$ Université de Paris, CNRS, Astroparticule et Cosmologie, F-75013 Paris, France.

${ }^{5}$ IFIC - Instituto de Física Corpuscular (CSIC - Universitat de València), c/Catedrático José Beltrán, 2, 46980 Paterna, Valencia, Spain. ${ }^{6}$ INFN, Sezione di Napoli, Complesso Universitario di Monte S. Angelo, Via Cintia ed. G, Napoli, 80126 Italy.

${ }^{7}$ Università di Napoli “Federico II", Dip. Scienze Fisiche "E. Pancini”, Complesso Universitario di Monte S. Angelo, Via Cintia ed. G, Napoli, 80126 Italy.

${ }^{8}$ INFN, Sezione di Roma, Piazzale Aldo Moro 2, Roma, 00185 Italy.

${ }^{9}$ Universitat Politècnica de Catalunya, Laboratori d'Aplicacions Bioacústiques, Centre Tecnològic de Vilanova i la Geltrú, Avda. Rambla Exposició, s/n, Vilanova i la Geltrú, 08800 Spain.

${ }^{10}$ NCSR Demokritos, Institute of Nuclear and Particle Physics, Ag. Paraskevi Attikis, Athens, 15310 Greece.

${ }^{11}$ INFN, Sezione di Genova, Via Dodecaneso 33, Genova, 16146 Italy.

${ }^{12}$ University of Granada, Dept. of Computer Architecture and Technology/CITIC, 18071 Granada, Spain.

${ }^{13}$ Friedrich-Alexander-Universität Erlangen-Nürnberg, Erlangen Centre for Astroparticle Physics, Erwin-Rommel-Straße 1, 91058 Erlangen, Germany.

${ }^{14}$ Universitat Politècnica de València, Instituto de Investigación para la Gestión Integrada de las Zonas Costeras, C/ Paranimf, 1, Gandia, 46730 Spain.

${ }^{15}$ Nikhef, National Institute for Subatomic Physics, PO Box 41882, Amsterdam, 1009 DB Netherlands.

${ }^{16}$ University Mohammed V in Rabat, Faculty of Sciences, 4 av. Ibn Battouta, B.P. 1014, R.P. 10000 Rabat, Morocco.

${ }^{17}$ INFN, Sezione di Bologna, v.le C. Berti-Pichat, 6/2, Bologna, 40127 Italy.

\footnotetext{
${ }^{1}$ also at Dipartimento di Fisica, INFN Sezione di Padova and Università di Padova, I-35131, Padova, Italy
} 
${ }^{18}$ Università di Bologna, Dipartimento di Fisica e Astronomia, v.le C. Berti-Pichat, 6/2, Bologna, 40127 Italy.

${ }^{19}$ KVI-CART University of Groningen, Groningen, the Netherlands.

${ }^{20}$ INFN, Laboratori Nazionali del Sud, Via S. Sofia 62, Catania, 95123 Italy.

${ }^{21}$ North-West University, Centre for Space Research, Private Bag X6001, Potchefstroom, 2520 South Africa.

${ }^{22}$ Instituto Español de Oceanografía, Unidad Mixta IEO-UPV, C/ Paranimf, 1, Gandia, 46730 Spain.

${ }^{23}$ University Mohammed I, Faculty of Sciences, BV Mohammed VI, B.P. 717, R.P. 60000 Oujda, Morocco.

${ }^{24}$ Subatech, IMT Atlantique, IN2P3-CNRS, Université de Nantes, 4 rue Alfred Kastler - La Chantrerie, Nantes, BP 2072244307 France.

${ }^{25}$ Università di Salerno e INFN Gruppo Collegato di Salerno, Dipartimento di Matematica, Via Giovanni Paolo II 132, Fisciano, 84084 Italy.

${ }^{26}$ ISS, Atomistilor 409, Măgurele, RO-077125 Romania.

${ }^{27}$ University of Amsterdam, Institute of Physics/IHEF, PO Box 94216, Amsterdam, 1090 GE Netherlands.

${ }^{28}$ TNO, Technical Sciences, PO Box 155, Delft, 2600 AD Netherlands.

${ }^{29}$ Università degli Studi della Campania "Luigi Vanvitelli", Dipartimento di Matematica e Fisica, viale Lincoln 5, Caserta, 81100 Italy.

${ }^{30}$ Università La Sapienza, Dipartimento di Fisica, Piazzale Aldo Moro 2, Roma, 00185 Italy.

${ }^{31}$ Università di Bologna, Dipartimento di Ingegneria dell'Energia Elettrica e dell'Informazione "Guglielmo Marconi", Via dell’Università 50, Cesena, 47521 Italia.

${ }^{32}$ Cadi Ayyad University, Physics Department, Faculty of Science Semlalia, Av. My Abdellah, P.O.B. 2390, Marrakech, 40000 Morocco.

${ }^{33}$ University of the Witwatersrand, School of Physics, Private Bag 3, Johannesburg, Wits 2050 South Africa.

${ }^{34}$ Università di Catania, Dipartimento di Fisica e Astronomia "Ettore Majorana", Via Santa Sofia 64, Catania, 95123 Italy.

${ }^{35}$ INFN, LNF, Via Enrico Fermi, 40, Frascati, 00044 Italy.

${ }^{36}$ INFN, Sezione di Bari, via Orabona, 4, Bari, 70125 Italy.

${ }^{37}$ International Centre for Radio Astronomy Research, Curtin University, Bentley, WA 6102, Australia.

${ }^{38}$ University of Bari, Via Amendola 173, Bari, 70126 Italy.

${ }^{39}$ University Würzburg, Emil-Fischer-Straße 31, Würzburg, 97074 Germany.

${ }^{40}$ Western Sydney University, School of Computing, Engineering and Mathematics, Locked Bag 1797, Penrith, NSW 2751 Australia.

${ }^{41}$ Università di Salerno e INFN Gruppo Collegato di Salerno, Dipartimento di Fisica, Via Giovanni Paolo II 132, Fisciano, 84084 Italy.

${ }^{42}$ Università di Genova, Via Dodecaneso 33, Genova, 16146 Italy.

${ }^{43}$ Normandie Univ, ENSICAEN, UNICAEN, CNRS/IN2P3, LPC Caen, LPCCAEN, 6 boulevard Maréchal Juin, Caen, 14050 France.

${ }^{44}$ University of Granada, Dpto. de Física Teórica y del Cosmos \& C.A.F.P.E., 18071 Granada, Spain.

${ }^{45}$ NIOZ (Royal Netherlands Institute for Sea Research), PO Box 59, Den Burg, Texel, 1790 AB, the Netherlands.

${ }^{46}$ Tbilisi State University, Department of Physics, 3, Chavchavadze Ave., Tbilisi, 0179 Georgia.

${ }^{47}$ Leiden University, Leiden Institute of Physics, PO Box 9504, Leiden, 2300 RA Netherlands.

${ }^{48}$ National Centre for Nuclear Research, 02-093 Warsaw, Poland.

${ }^{49}$ Institut Universitaire de France, 1 rue Descartes, Paris, 75005 France.

${ }^{50}$ University of Johannesburg, Department Physics, PO Box 524, Auckland Park, 2006 South Africa.

${ }^{51}$ Eberhard Karls Universität Tübingen, Institut für Astronomie und Astrophysik, Sand 1, Tübingen, 72076 Germany.

${ }^{52}$ Università di Pisa, Dipartimento di Fisica, Largo Bruno Pontecorvo 3, Pisa, 56127 Italy.

${ }^{53}$ Laboratoire Univers et Particules de Montpellier, Place Eugène Bataillon - CC 72, Montpellier Cédex 05, 34095 France.

${ }^{54}$ Friedrich-Alexander-Universität Erlangen-Nürnberg, Remeis Sternwarte, Sternwartstraße 7, 96049 Bamberg, Germany.

${ }^{55}$ Université de Haute Alsace, 68100 Mulhouse Cedex, France.

${ }^{56}$ University of Münster, Institut für Kernphysik, Wilhelm-Klemm-Str. 9, Münster, 48149 Germany.

${ }^{57}$ Utrecht University, Department of Physics and Astronomy, PO Box 80000, Utrecht, 3508 TA Netherlands.

${ }^{58}$ INFN, CNAF, v.le C. Berti-Pichat, 6/2, Bologna, 40127 Italy. 